\title{
Mechanisms of Degradation in Adhesive Joint Strength: Glassy Polymer Thermoset Bond in a Humid Environment
}

\author{
Jamie M. Kropka, ${ }^{*}$ Douglas B. Adolf, ${ }^{*}$ Scott Spangler, \\ Kevin Austin, ${ }^{*}$ and Robert S. Chambers ${ }^{\dagger}$ \\ * Materials Sciences and Engineering Center \\ Sandia National Laboratories, Albuquerque, NM 87185-0958 \\ ${ }^{\dagger}$ Engineering Sciences Center \\ Sandia National Laboratories, Albuquerque, NM87185-0346
}

\begin{abstract}
$\underline{\text { Abstract }}$
The degradation in the strength of napkin-ring (NR) joints bonded with an epoxy thermoset is evaluated in a humid environment. While adherend composition (stainless steel and aluminum) and surface preparation (polished, grit blasted, primed, coupling agent coated) do not affect virgin (time $=0$ ) joint strength, they can significantly affect the role of moisture on the strength of the joint. Adherend surface abrasion and corrosion processes are found to be key factors in determining the reliability of joint strength in humid environments. In cases where surface specific joint strength degradation processes are not active, decreases in joint strength can be accounted for by the glass transition temperature, $T_{\mathrm{g}}$, depression of the adhesive associated with water sorption. Under these conditions, joint strength can be rejuvenated to virgin strength by drying. In addition, the decrease in joint strength associated with water sorption can be predicted by the Simplified Potential Energy Clock (SPEC) model by shifting the adhesive reference

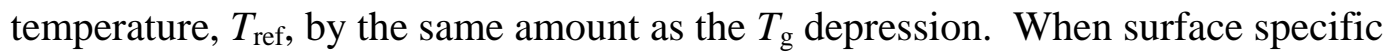
degradation mechanisms are active, they can reduce joint strength below that associated with adhesive $T_{\mathrm{g}}$ depression, and joint strength is not recoverable by drying. A critical relative humidity (or, potentially, critical water sorption concentration), below which the surface specific degradation does not occur, appears to exist for the polished stainless steel joints.
\end{abstract}

Address correspondence to Jamie M. Kropka, Materials Sciences and Engineering Center, Sandia Corporation, Albuquerque, NM 87185-0985, USA. E-mail: jmkropk@sandia.gov

Keywords: adhesion, degradation, humidity, mechanism, viscoelasticity 


\section{Introduction}

The performance and reliability of many electrical, mechanical and optical assemblies depend on the integrity of adhesively bonded joints. Unfortunately, the ability to predict the performance of critical polymer-solid interfaces is limited. For instance, cohesive zone modeling techniques for crack growth along a polymer-solid interface typically do not treat the polymer as a non-linear viscoelastic (NLVE) material in a physics-based manner. Without this, nonlinear relaxations ${ }^{1}$ and history dependencies ${ }^{2}$ that can have a significant impact on how failure occurs may not be accounted for. At the same time, interfacial failure analyses using a $\mathrm{NLVE}^{3}$ representation of the adhesive, which capture nonlinear relaxation and history dependencies, have difficulty defining a failure metric when there are severe strain gradients in the problem, such as at sharp corners. It should be noted that these limitations exist for a freshly bonded, virgin joint. Addressing the reliability of interfacial bonds over time presents even further challenges.

While all of these limitations cannot likely be addressed in a single effort, steps must continue to be made in developing the understanding and tools necessary to predict the performance of polymer bonded interfaces. Over the years, many approaches have been taken to understand adhesion and adhesive joint failure, much of which has been reviewed. ${ }^{4}$ Therefore, it is necessary to clearly define the purpose of the current investigation so that the results can be fit into the context of this history. Attempts have been made to understand and predict both the propagation of existing surface cracks ${ }^{5}$ and the critical traction defining the initiation of de-bonding in an as-designed, un-cracked geometry, be it under relatively uniform stress loadings ${ }^{6}$ or where severe stress gradients exist within the joint. ${ }^{7}$ The current study addresses the problem of assessing the ability of an adhesive bond with no known defects to survive an applied load. The goal is not only to predict the critical traction of a virgin bond, but also to define how that strength changes under environmental exposures. There are certainly many environmental conditions that may affect the strength of a polymer-solid interface, e.g., thermal and strain histories, exposure to small molecule absorbents, etc. Here, we focus on the change of adhesive strength in humid environments, but the testing framework established enables investigations into other environmental influences as well.

The complexity of an adhesively bonded joint makes predicting how it will behave over long periods of time a challenging proposition. For example, failure mechanisms can change with variables such as joint construction and joint history. Considering polymer adhesives bonding metal adherends, the polymer is often the weaklink in the structure if weak boundary layers ${ }^{8}$ are not present. Because of this, work focused on what occurs in the polymer adhesive and how this might be used to predict failure of the bonded joint is of particular interest. In this manuscript, a combination of carefully designed experiments and accompanying finite element stress analyses, with a NLVE representation of the polymer adhesive, are used to resolve adhesive joint failure 
mechanisms with the ultimate goal of developing a computational approach to predict failure. The challenges of severe strain gradients in the joint are avoided by intelligent design of the test geometries. Two geometries that fit this criterion include the napkinring $^{2}$ (NR) and the saucer. ${ }^{6}$ In this work, only the napkin-ring joint geometry is used. While the napkin-ring is limited to resolving critical shear tractions, the short diffusion path for moisture through the adhesive makes it attractive for realizing the effects of humid environments on joint strength in relatively short periods of time. The effect of moisture on polymer adhesion is a subject that has received considerable attention over many years, with work focused on both practical and theoretical aspects. The goal of this manuscript is not to review those findings, that has been done elsewhere. ${ }^{9}$ Rather, the focus will be maintained on a new set of results, and previous findings will be leveraged to help interpret the new observations where appropriate. The use of NLVE models to represent the adhesive distinguishes this work from damage mechanics based approaches. ${ }^{9}$ NLVE enables tracking of history effects through a "material clock". 3,10 The potential of this history tracking to account for time evolution of joint strength is of particular interest for long-term prediction capability. The current work set out to address a number of questions for a glassy polymer thermoset bonding the NR. The first was to determine how adherend composition and surface preparation affect the reliability of interfacial bonds over time in humid environments. The results from initial experiments inspired further investigations aimed at answering whether the absorption of water into the adhesive could completely account for the changes in bond strength or whether other factors must be accounted for. Then, both practical application drivers and the desire to further understand the mechanism of joint strength degradation led to experiments designed to resolve whether joint strength could be rejuventated to its virgin value. Initial examinations into the role of relative humidity $(\mathrm{RH})$ on joint strength depression were also carried out. Here, the interest was in determining whether RH just defined the saturation concentration of water absorbed by the adhesive or whether there might be some critical RH level, or critical water sorption concentration, that must be achieved before significant changes in bond strength are resolved. ${ }^{11}$ The results of these investigations and current interpretations of the results will be addressed in the following sections of this manuscript.

\section{Experimental}

The napkin-rings were machined from either 304 stainless steel (304SS) or 6061T6 aluminum (Al) with adherend outer diameter of $25.4 \mathrm{~mm}$ and annulus dimensions: inner diameter of $16.5 \mathrm{~mm}$, outer diameter of $19.1 \mathrm{~mm}$, and height of $1.3 \mathrm{~mm}$. A schematic of the geometry is shown in Figure 1(a). 


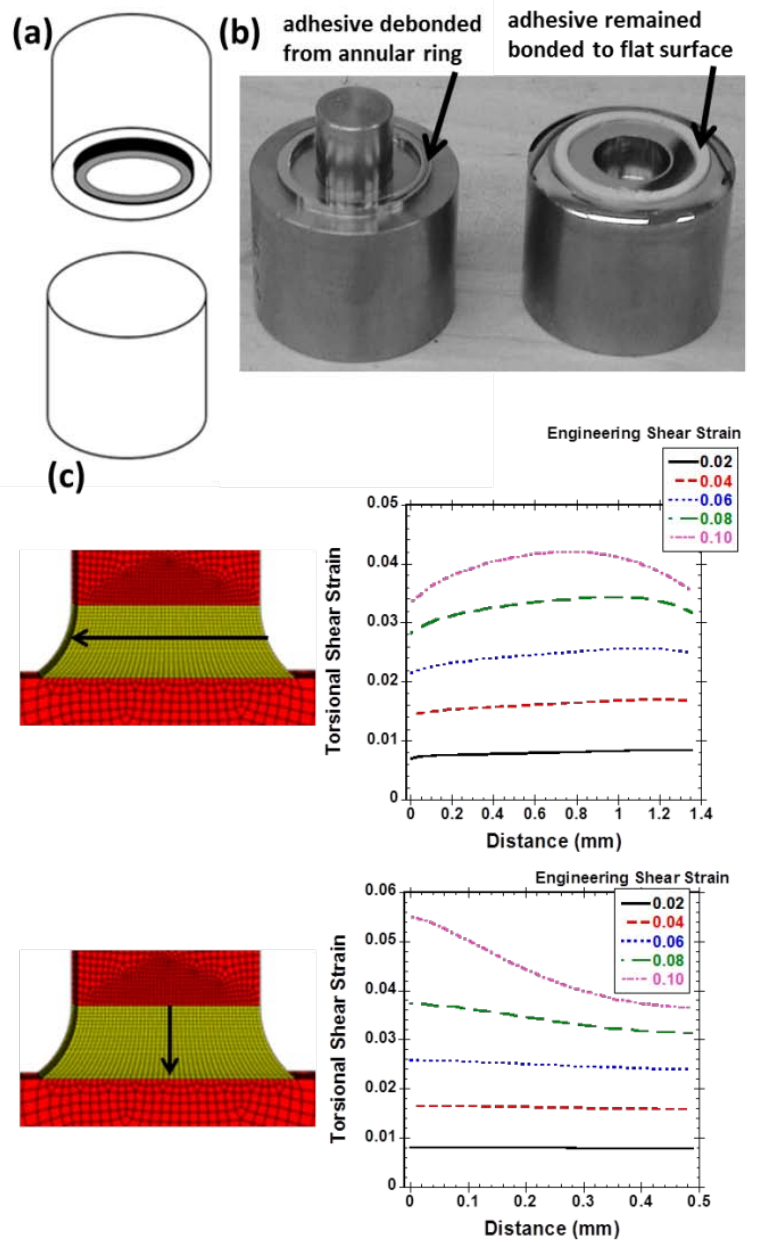

Fig. 1: (a) Schematic of NR geometry, (b) image of NR after failure and (c) shear strain distributions within the adhesive during joint loading. Torsional Shear Strain $=1 / 2$ (Engineering Strain).

Multiple surface finishes for the annuli and flat plug were examined, including the following: (1) polished (smooth), (2) blasted (rough) with 60 grit red garnet (Barton Mines, 200 microns), (3) blasted with \#39 soda lime glass beads (Crystal Mark, 50 microns), (4) coated with a chromate primer, BR 127 (Cytek) and (5) coated with a silane coupling agent, (3-Glycidoxypropyl)trimethoxysilane, GPS (GELEST). All blasting was performed using a Swam-Blast MV-21 (Crystal Mark). Micrographs of the surfaces are given in Figure 2. An Axio CSM 700 confocal microscope (Zeiss) resolved surface roughness, reported as the average absolute value of the height, $R_{\mathrm{a}}$, as (a) 0.197 $\mu \mathrm{m}$, (b) $2.02 \mu \mathrm{m}$, (c) $0.085 \mu \mathrm{m}$, (d) $2.78 \mu \mathrm{m}$ and (e) $0.36 \mu \mathrm{m}$. 


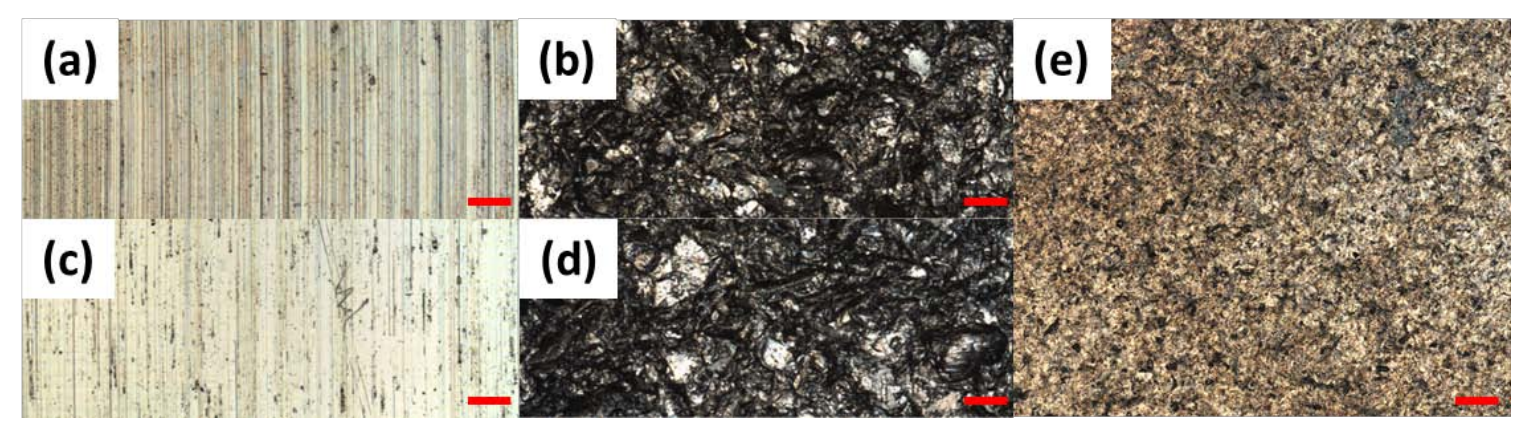

Fig. 2: Surface micrographs of (a) polished 304SS, (b) red garnet blasted 304SS, (c) polished $\mathrm{Al}$, (d) red garnet blasted $\mathrm{Al}$ and (e) glass bead blasted 304SS. Red lines on lower part of micrograph represent 50 $\mu \mathrm{m}$.

The primer was deposited with a flexible foam swab using a light contact force between the swab and application surface. To prevent pooling of the primer and attain an even coating thickness, the application surface was kept horizontal and the swab covered the entire application area. After application, the primer was air cured for 30 minutes and post-cured for 30 minutes at $120^{\circ} \mathrm{C}$. The primer thickness was $3 \mu \mathrm{m}$ as measured by a Dektak 3 Surface Profilometer (Veeco), within the manufacturer suggested range 2.5 to $7.5 \mu \mathrm{m}$. The glass transition temperature of the primer processed in this manner was measured on a Q2000 differential scanning calorimeter (TA Instruments) to have a midpoint of $115^{\circ} \mathrm{C}$ during a heating cycle at $10^{\circ} \mathrm{C} / \mathrm{min}$. The silane coupling agent was added at a level of $2 \mathrm{wt} \%$ to a $95 \mathrm{wt} \%$ ethanol/ $5 \mathrm{wt} \%$ water solution adjusted to $\mathrm{pH} 4.5$ 5.5 with acetic acid. A minimum of five minutes was allowed for hydrolysis and silanol formation, after which the adherends were dipped into the solution, agitated gently, and removed after 1-2 minutes. Subsequently, adherends were dipped into ethanol to rinse any excessive deposited material and then cured for 24 hours at room temperature.

Before applying any coatings or bonding the joints, surfaces were cleaned in solvent filled ultrasonic baths (acetone then isopropanol) and blown dry with nitrogen. The adherends were bonded together with the annulus as the upper surface, and the bond line $(0.5 \mathrm{~mm})$ was defined by a steel dowel and set-screw that could be backed off after cure to allow frictionless testing. Adhesive wetting of the adherend annulus is controlled by not submersing the annulus into the liquid adhesive, but initiating contact between the annulus and adhesive and then rotating the annulus to enable intimate surface contact. Wetting at the flat adherend is not controlled, but finite element analyses show that adhesive "yielding" in the macroscopic joint is not sensitive to the details of the wetting profile at this surface. Just after manufacture, all joints were annealed above $T_{\mathrm{g}}$ for 30 minutes and then cooled at $0.5^{\circ} \mathrm{C} / \mathrm{min}$ to room temperature to establish a known history for the viscoelastic adhesive.

After preparation and annealing, some napkin-ring joints were exposed to $60^{\circ} \mathrm{C}$ and a controlled relative humidity (RH) environment prior to mechanical testing. A bell 
jar containing de-ionized water, sealed with vacuum grease and vented to the oven, was used to maintain the $100 \% \mathrm{RH}$ environment. A Z-Plus humidity controlled chamber (Cincinnati Sub-Zero) was used to attain other RH levels. After reaching an apparent steady state strength in the humid environment, some joints were also dried at $60^{\circ} \mathrm{C}$ in a desiccated atmosphere.

All joint mechanical loading was completed as a controlled displacement torsional ramp ( 2\% strain/sec) on an Instron 55MT torsional test frame at $T=23^{\circ} \mathrm{C}$. A minimum of three joints were evaluated at each condition to establish the average stress at failure reported. Error bars denote one standard deviation of the measurements, except for instances where only three joints were evaluated. Maximum and minimum measured stress at failure were used as error bars where only three samples were tested. In the case of aged joints, mechanical testing occurred as soon as possible (within an hour) after removal from the aging condition and equilibrating to the test temperature. For adherends constructed from identical material, de-bonding occurred preferentially at the annulus [see Figure 1(b)] due to the small meniscus formed at the lower, flat adherend surface (thereby creating a somewhat larger bonding area). In addition, computational analyses have shown the stresses and strains within the adhesive during torsional loading of the napkin-ring geometry to be uniformly distributed [see Figure 1(c)], which enables an experimental determination of the critical shear traction at the point of joint failure. In Figure 1(c) the yellow elements represent the adhesive and the red elements represent the adherends, with the annular ring at the top of the adhesive. The arrows illustrate the trace for which local strains are plotted to the right, with the point of the arrow at the largest distance. In these analyses, the cross-section of the adhesive bondline was constructed to match that directly measured from tested joints. Analyses of annular adhesive bondlines with 90 degree corners at the bonding interfaces give the same maximum in the macroscopic joint torsional shear stress versus torsional shear strain response (a measure of the adhesive "yield"). Thus, identification of this "yielding" point is not noted to be sensitive to bondline geometry.

The adhesive used for testing consisted of the diglycidyl ether of bisphenol A (EPON ${ }^{\circledR}$ Resin 828, Momentive) cured with diethanolamine (DEA, Fisher Scientific) at 12 parts per hundred resin (phr) and hence referred to as 828/DEA. This system exhibits a $T_{\mathrm{g}}$ that has a midpoint of $70^{\circ} \mathrm{C}$ when cured for 24 hours at $70^{\circ} \mathrm{C}$. In addition to using the adhesive to bond napkin-ring joints, thin disk samples, diameter $(d) \sim 1 \mathrm{~cm}$ and thickness (l) $160 \mu \mathrm{m}$, of the adhesive were also used to quantify water absorption within the material using a Q5000 SA dynamic vapor sorption analyzer (TA Instruments). To do this, samples were first dried at $T=60^{\circ} \mathrm{C}$ and $\mathrm{RH}=0 \%$ to remove residual water from the manufacturing process and then cycled through multiple sorption and desorption cycles. The $d / l$ ratio of approximately 60 enables a 1-D diffusion approximation and evaluation of the diffusion coefficient of water through the adhesive. The $T_{\mathrm{g}}$ of these water saturated adhesive discs was also evaluated on a Q2000 differential scanning calorimeter (TA 
Instruments) in a heat-cool-heat routine between $-50^{\circ} \mathrm{C}$ and $150^{\circ} \mathrm{C}$ at $10^{\circ} \mathrm{C} / \mathrm{min}$. The $T_{\mathrm{g}}$ was evaluated from the heating cycle, taking into account data from the first and second heating cycles. A second adhesive, EPON® Resin 828 (Momentive) cured with Jeffamine ${ }^{\circledR}$ T-403 polyetheramine (Huntsman) at 43 parts per hundred resin, was also used for the tests represented in Figure 4. This material is referred to as 828/T403 and exhibits a $T_{\mathrm{g}}$ that has a midpoint of $85^{\circ} \mathrm{C}$ when fully cured.

All stress analyses were performed using the ADAGIO finite element code (inhouse software developed at Sandia National Laboratories, NM). ADAGIO is a threedimensional implicit quasi-statics and dynamics code with a versatile element library, nonlinear material models, large deformation capabilities, and contact. It is built on the SIERRA framework, providing an efficient data management framework for parallel computing. Fully three-dimensional finite element models were built using eight-node uniform strain elements generated with the Cubit meshing software. Meshes were constructed to have twenty elements through the radial width and ten elements through the thickness of the epoxy joint. The ADAGIO solutions were obtained through a conjugate gradient algorithm that enforces the momentum balance by minimizing the force residuals. Convergence was defined by requiring the relative residual tolerance to be less than or equal to $1.0 \times 10^{-6}$ (based on the ratio of the net residual to the L2 norm of the total reactive force).

Because epoxies are viscoelastic materials with fading memory, it is important to capture their evolving history. Thus all analyses reproduced the history of the experiments as faithfully as possible. Calculations assumed a stress-free state at the annealing temperature of $75^{\circ} \mathrm{C}$. Samples then were cooled to the test temperature of $23^{\circ} \mathrm{C}$ at $0.5^{\circ} \mathrm{C} / \mathrm{min}$, and the thermal residual stresses and strains were computed to define the initial conditions for the imposed mechanical loading. A torsional strain ( $2 \%$ epoxy shear/sec) was then imposed by kinematically specifying a prescribed rotation on a node set at the free end of one of the NR cylinders while rotationally constraining the end of the opposing cylinder. Joint geometry was idealized, representing the adhesive as a simple annular region with 90 degree corners at the bonding interfaces and a $0.5 \mathrm{~mm}$ bondline thickness, $h$. Later calculations have demonstrated only minor sensitivities to joint geometry, which do not affect the conclusions drawn herein. The 828/DEA adhesive was represented with a nonlinear viscoelastic constitutive equation, (the Simplified Potential Energy Clock (SPEC) model) using the material parameters defined previously. ${ }^{10}$ A brief summary of the key aspects of the SPEC model is given in the Appendix. The 304 stainless steel cylinders were modeled with a Young's modulus, yield strength and Poisson's ratio value of $200 \mathrm{GPa}, 273 \mathrm{MPa}$ and 0.275 , respectively. The 304 stainless steel did not yield and hence acted elastically in the calculations. The coefficient of thermal expansion of the 304 stainless steel was taken to be $17.3 \mathrm{ppm} /{ }^{\circ} \mathrm{C}$. 


\section{Results and Discussion}

The influence of adherend composition and surface preparation on the reliability of adhesive joint strength was first examined by preparing both 304SS and Al napkinring joints with multiple surface preparations and exposing the joints to a $100 \% \mathrm{RH}$ environment at $60^{\circ} \mathrm{C}$. The control was in a dry environment at $\mathrm{T}=60^{\circ} \mathrm{C}$. The $60^{\circ} \mathrm{C}$ environment was chosen such that water diffusion into the joint, or any type of activated degradation process, may proceed at an adequate rate while keeping the bonding adhesive below its glass transition temperature, $T_{\mathrm{g}}$. Joints were periodically removed from this environment, cooled to room temperature, and then a ramped (displacement controlled) torsional load was applied to failure. The room temperature shear stress at failure is given as a function of exposure time to $100 \% \mathrm{RH}$ at $60^{\circ} \mathrm{C}$ in Figure 3. 


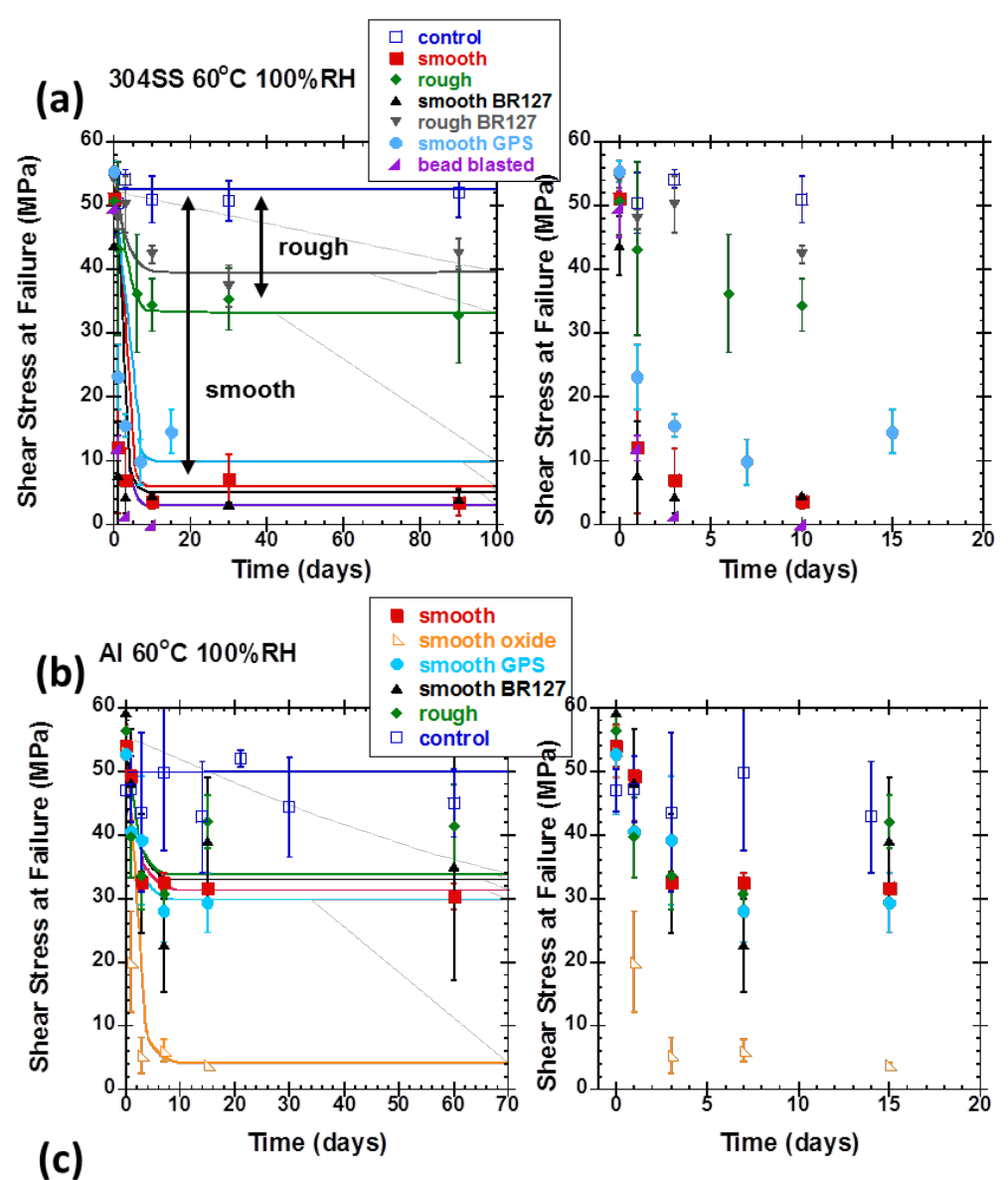

(c)
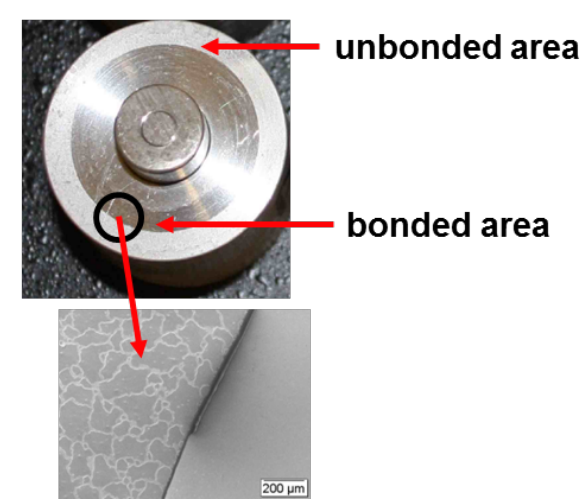

Fig. 3: The time dependence of the shear stress at failure of napkin-ring joints formed from (a) 304SS and (b) Al adherends with various surface preparations. The lines are just a guide to the eye. Plots to the right illustrate the short time behavior of the data. "Smooth oxide" refers to polished adherends that exhibited corrosion at the $\mathrm{Al}$-adhesive bonding interface during the environmental exposure. An example of this corrosion on the unbonded area of the joint is given in (c). 
In Figure 3(a), results are provided for the 304 SS adherends with a number of different surface preparations. The first observation from this plot is that the virgin joint shear stress at failure (evaluated at time, $t=0$ ) is independent of surface preparation, even when comparing a polished surface (smooth) to a grit blasted surface (rough). While the independence of joint strength on surface composition and preparation has been reported in tests designed to resolve the critical traction at initiation of joint failure, ${ }^{2}$ this is not a universal finding. Indeed, many reports attribute an increase in joint strength with surface roughening to increased bonding area and a "mechanical interlocking” phenomena. ${ }^{12}$ Granted, one might anticipate a trade-off between these contributions and stress concentrations, which would exist at sharp features on the rough interface, when it comes to determining how the strength of an interface would change with roughening if a local stress-based criterion for failure is assumed. This trade-off, along with other potential explanations for changes in joint strength associated with surface roughening have been discussed elsewhere ${ }^{6}$ and will not be further examined here. For the napkinring joint, the independence of virgin joint strength on surface preparation may well be associated with the mechanism of failure in the joint. The temperature dependence of the experimental shear stress at failure for the napkin-ring joint coincides with the temperature dependence of the predicted shear "yield" stress of the adhesive, ${ }^{6}$ where polymer adhesive viscoelastic relaxation rates increase to the point that stress decays faster than incremented by the loading ramp in what has been termed "run-away" nonlinear viscoelasticity. ${ }^{2}$ Figure 4 further illustrates the correlation, with multiple adhesives and multiple methods of predicting shear yield. Specifically, predictions in Figure 4 are associated with (1) the adhesive shear yield stress evaluated under a simple shear loading in a single element analysis and (2) the maximum stress sustained in a napkin-ring joint under a torsional load. 


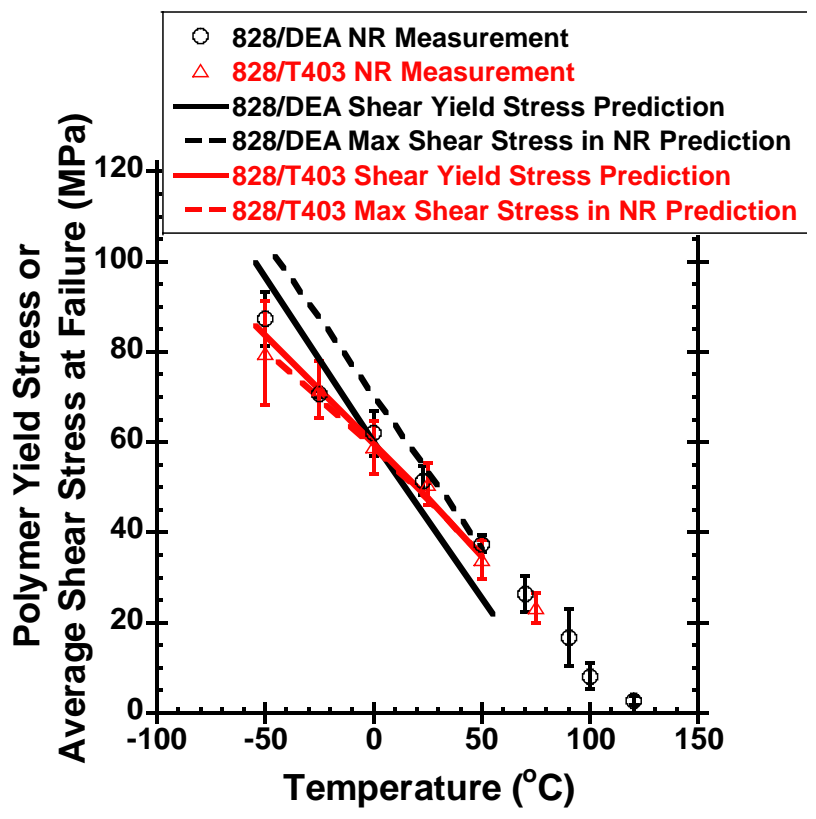

Fig. 4: Experimental shear stress at failure versus temperature for virgin napkin-ring joints bonded with 828/DEA and 828/T403, plotted along with two SPEC predictions described in the text.

This implies that failure of the joint may be initiated by cohesive failure within the adhesive and this could explain the insensitivity of joint strength to surface preparation. If the interfacial shear strength is greater than the cohesive shear strength of the adhesive, then joint strength will remain independent of surface composition and preparation unless the changes reduce the interfacial strength below that of the cohesive strength of the adhesive.

Moving on to the time dependence of the shear stress at failure in Figure 3(a), note that the control samples, joints with polished adherends that were exposed to temperatures of $60^{\circ} \mathrm{C}$ in a desiccated environment, demonstrated no significant change in the shear stress at failure over the three month test period for which they were evaluated. Given that the exposure temperature was close to, but below, the $T_{\mathrm{g}}$ of the adhesive material, physical aging ${ }^{13}$ of the adhesive is likely to be significant in these environments. The volume relaxation of the adhesive to a more dense state associated with the physical aging process can result in higher residual stress build-up in the adhesive since confinement at the bonded interfaces does not allow the adhesive to contract as it would like. The volume relaxation process also slows relaxation rates of the adhesive. Any such effects either cancel each other out or do not have a significant impact on the strength of the joint over the 90 days tested. On the other hand, all joints that experienced the $100 \%$ RH appear to equilibrate to a shear stress at failure less than that of the virgin joints. Since the control samples did not exhibit this behavior, the depressed joint strength can be associated with the presence of moisture, not elevated temperature. 
Further, the magnitude of the equilibrated joint strength is strongly affected by the surface abrasion of the 304 SS adherends. Joints blasted with the red garnet media exhibited equilibrated strengths on the order of $~ 30-40 \mathrm{MPa}$, with or without the BR127 primer (the primer was placed on these joints as an exploratory experiment, not because 304SS was anticipated to corrode over the timescale of the experiment). This is significantly below the 50 MPa strength of the virgin joints but well above the equilibrated strengths of the smooth surface joints, $\sim 5-10 \mathrm{MPa}$, with or without the BR127 primer and GPS silane coupling agent. Previous work ${ }^{11}$ has described abrading aluminum alloy adherends in anticipation of getting rapid changes in joint strength upon exposure to humid environments due to a known poor durability of sandblasted joints. This observation does not seem to hold for the 304SS joints tested here, as the abraded joints appear more durable.

The distinction of the dependence of equilibrated joint strength on surface abrasion led to the testing of an additional surface roughening technique, glass bead blasting. Curiously, this surface preparation gave approximately the same result as the smooth, polished surfaces [see Figure 3(a)]. This raises the question as to what the key factor in determining the equilibrated strength is. Could it be surface topology, surface chemistry (which could change if blast media remains embedded in the adherend surface), or some combination of multiple effects? Certainly the average absolute value of the height, $R_{\mathrm{a}}$, of the bead blasted surface $(0.36 \mu \mathrm{m})$ is closer to that of the polished adherends $(0.197 \mu \mathrm{m})$ than the red garnet blasted adherends $(2.02 \mu \mathrm{m})$. Maybe this implies a critical $R_{\mathrm{a}}$ necessary to achieve an increase in joint durability? Consider the case in which water induces a weak interfacial layer of the adhesive near the adherend (e.g., due to a local excess water concentration). In this scenario, how the adherend surface roughness height compares to the thickness of the weakened adhesive layer can impact the joint strength under a shear load. An ideally smooth surface would readily allow failure along the plane of the weak adhesive layer. On the other hand, when surface roughness height is much greater than weak adhesive layer thickness, failure of the joint would necessitate traversing both the weak and the bulk adhesive and hence mitigate the effects of the "weak boundary layer" on joint strength. Finite element analyses of simplistic geometries and scoping parameters for the weakened adhesive layer have illustrated this effect of surface roughness on predicted joint strength. ${ }^{14}$ However, while this physical mechanism may fit well with the results from the 304SS adherend joints, later in this manuscript conditions will be shown under which similar effects of surface roughness are not observed. This suggests a lack of applicability, or at least a lack of generality, of the mechanism and/or a role of other factors in determining joint durability. The surface topology of the adherends is also distinct between the two different blasting media (see Figure 5). Red garnet blasting results in sharper surface features than that of glass bead blasting. Going forward, the details of the interplay between surface topology and surface chemistry on the durability of these joints remains 
an area of interest and continues to be investigated. Later in this manuscript, the equilibrated strengths for the 304SS adherend joints will be further discussed. But at this point it is already clear that while surface preparation does not have an impact on the virgin strength of the joint, it does have an impact on the equilibrated strength of the joint upon exposure to humid environments.

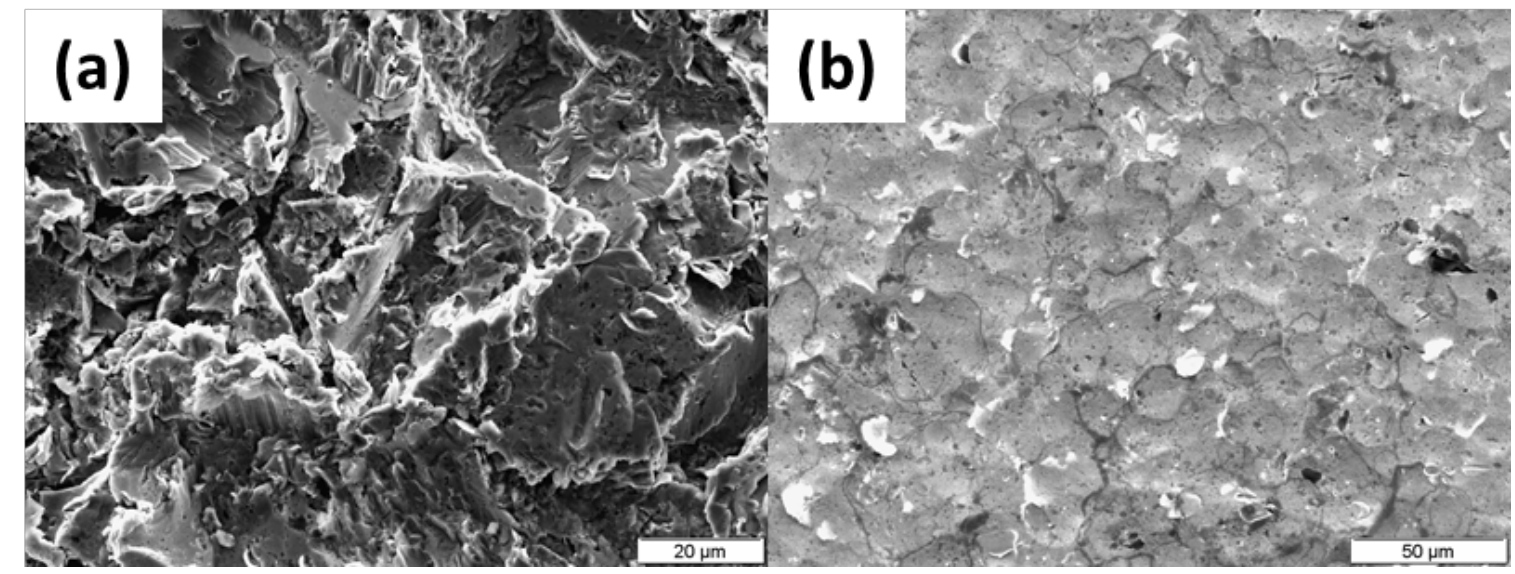

Fig. 5: Scanning electron microscopy (SEM) surface micrographs of (a) red garnet blasted 304SS and (b) glass bead blasted 304SS. Secondary electron images were obtained with a XL 40 environmental SEM (FEI).

Now focusing on Figure 3(b), where joints constructed from Al adherends are tested in much the same way as the joints constructed of 304 SS adherends in Figure 3(a), some similar and some different observations are made for the time dependence of joint strength in a humid environment $\left(T=60^{\circ} \mathrm{C}\right.$ and $\left.100 \% \mathrm{RH}\right)$. Once again, the virgin joint shear stress at failure is independent of surface preparation and indistinguishable from that of the 304 SS adherend joints. This finding further supports failure of the joints initiating cohesively within the polymer adhesive. The time dependent data demonstrated more scatter for the $\mathrm{Al}$ adherends than it did for the $304 \mathrm{SS}$ adherends. The root cause of this is not currently known, although many speculations could be made. For now, some statements on the data will be made despite the scatter. The control samples, joints with polished adherends that were exposed to $60^{\circ} \mathrm{C}$ in a desiccated environment, demonstrated no significant change in the shear stress at failure over the test period. All joints that experienced the $100 \% \mathrm{RH}$ equilibrated to a shear stress at failure less than that of the virgin joints. But for the $\mathrm{Al}$ adherends, only joints that exhibited a visible "corrosion" layer growing between the adherend and adhesive equilibrated to strengths of $\sim 5-10$ $\mathrm{MPa}$. Some pictures of the corrosion layer growth from these joints are given in Figure 3(c), but no further characterization of this layer has been done to this point. It should be noted that all joints exhibited this growth layer on the Al surface. However, in many cases the epoxy adhesive acted as a primer and prevented corrosion at the bond-line. 
Bond-line corrosion appears to be necessary to depress joint strength to the $~ 5-10 \mathrm{MPa}$ level. Humid environments have been shown to convert the oxide layer on $\mathrm{Al}$ to an $\mathrm{Al}$ hydroxide, which adheres poorly to the metal substrate ${ }^{15}$ and leads to a weak boundary layer in the joint. ${ }^{8}$ Such a weak boundary layer may account for the loss of strength observed in the corroded bond-line joints. All other joints equilibrated to strengths of $\sim 30-40 \mathrm{MPa}$, and there was no clear distinction between rough and smooth surfaces as there was for the 304SS joints. This brings into questions the potential mechanism of increased joint strength durability associated with surface roughness proposed above for the 304SS joints, or at least questions the generality of this mechanism. Certainly, factors such as water concentration at the interface and thickness of a weakened interfacial adhesive layer could vary between the adherends types due to differences in thermodynamic drivers. Assessments of how these parameters change with adherend type would need to be made in order to define whether they can account for the varying influence of surface roughness on joint durability in humid environments.

During exposure to $T=60^{\circ} \mathrm{C}$ and $100 \% \mathrm{RH}$, the adhesive absorbs water that acts as a plasticizer. To quantify these effects, thin discs of the adhesive were used to track water sorption into the polymer and to evaluate the effect of water sorption on the glass transition of the polymer. The results from these experiments at a number of relative humidity levels at $T=60^{\circ} \mathrm{C}$ are given in Figure 6 . 


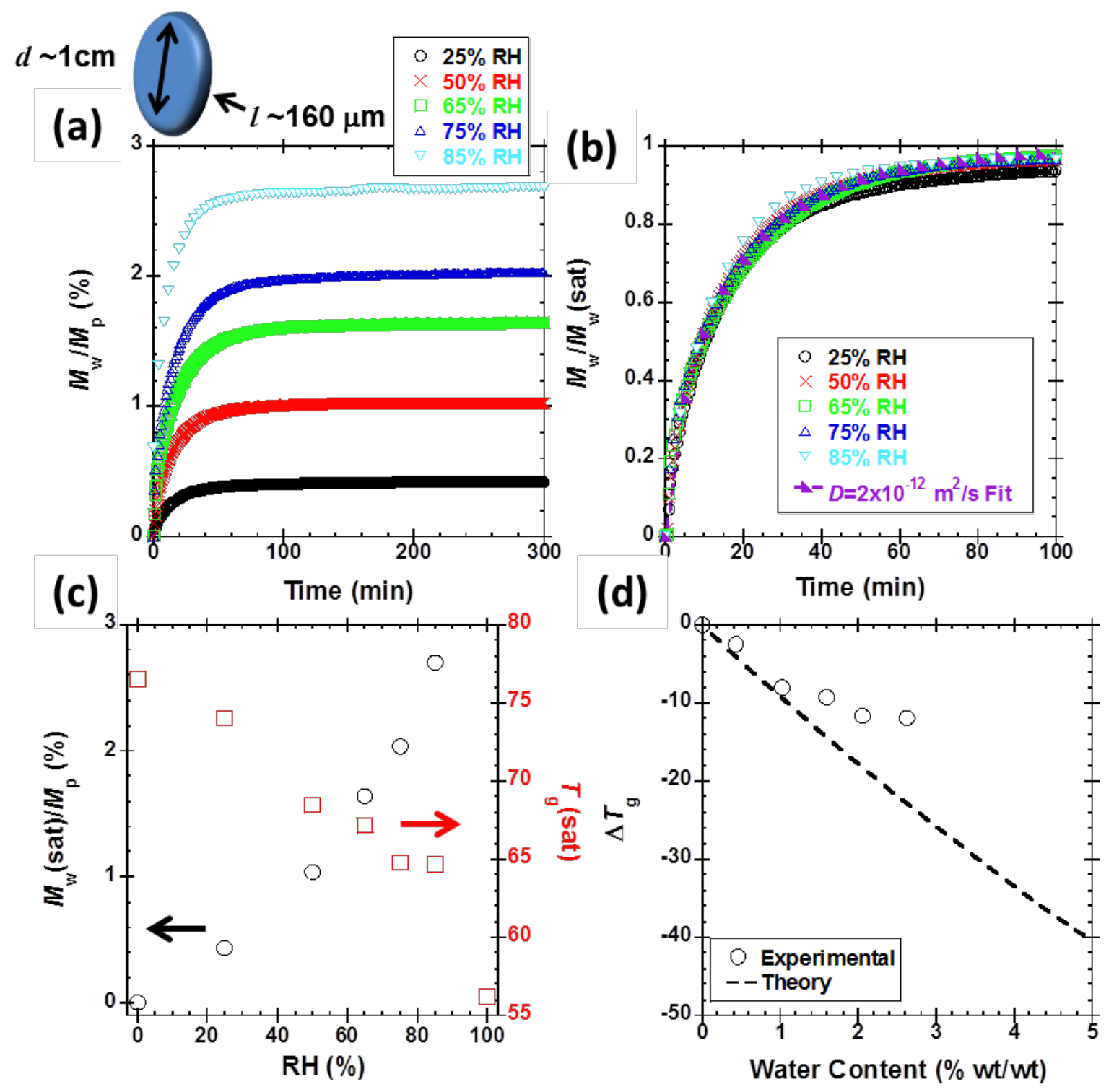

Fig. 6: (a) Adhesive sample geometry and water sorption into the 828/DEA polymer as a function of time at multiple RH, (b) normalization of water sorption by saturation level and extraction of a diffusion coefficient, (c) water saturation level and adhesive $T_{\mathrm{g}}$ at saturation as a function of $\mathrm{RH}$ and (d) comparison of $T_{\mathrm{g}}$ depression measurements to theoretical ${ }^{16}$ predictions.

From Figure 6(a), where water sorption is tracked as the ratio of the mass of water, $M_{\mathrm{w}}$, to the mass of polymer, $M_{\mathrm{p}}$, both the rate at which water is absorbed and the equilibrium water sorption reached are evident. While not shown, subsequent desorption and absorption cycles on the same sample were equivalent, and hence no irreversible processes have been observed in these tests. Since 1-D diffusion is applicable in these 
large aspect ratio samples, a water diffusion coefficient, $D$, through the polymer can be determined from fits to the 1-D Fickian diffusion solution given in Equation 1, ${ }^{17}$

$$
\frac{M_{w}(t)}{M_{w}(s a t)}=1-\frac{8}{\pi^{2}} \sum_{k \text { odd }} \frac{e^{-t D\left(\frac{k \pi}{l}\right)^{2}}}{k^{2}} \cong 1-\exp \left[-7.3\left(\frac{D t}{l^{2}}\right)^{0.75}\right] \quad \text { Eq. } 1
$$

where $M_{\mathrm{w}}(t)$ is the mass of water absorbed into the polymer at time $t, M_{\mathrm{w}}(\mathrm{sat})$ is the mass of absorbed water at saturation, and $l$ is the polymer thickness in the diffusion direction. $D$ is found to be $2 \times 10^{-12} \mathrm{~m}^{2} / \mathrm{s}$ [see Figure 6(b)]. This value is independent of RH and comparable to that observed for water diffusion through other thermoset epoxies. ${ }^{18}$ It should be noted that the bulk sorption rate suggests that the napkin-ring joint (assuming 1-D diffusion in the radial direction with a diffusion length, $L$, of $\sim 0.63 \mathrm{~mm}$ ) would be water saturated in approximately 3 days $\left(\tau \sim L^{2} / D\right)$, which coincides well with the time it took napkin-ring joint strength to equilibrate (see Figure 3 ). This could mean that accounting for bulk diffusion may be sufficient (i.e., it may not be necessary to address any potential differences in interfacial diffusion rates) to describe water sorption effects on joint strength. This hypothesis could be tested more rigorously by acquiring more data on napkin-ring joint strength decay within the first three days of exposure to humid environments.

Figure 6(c) shows both the saturated water concentration within the polymer adhesive and the bulk polymer $T_{\mathrm{g}}$ at saturation as a function of the RH level at $T=60^{\circ} \mathrm{C}$. As anticipated, the absorbed water acts as a plasticizer and depresses the $T_{\mathrm{g}}$ of the bulk polymer. The magnitude of the $T_{\mathrm{g}}$ depression increases with the amount of water absorbed in the polymer. The change in $T_{\mathrm{g}}$ observed with water sorption is compared to theory ${ }^{16}$ in Figure 6(d). The theory predicts the $T_{\mathrm{g}}$ of the water imbibed polymer to evolve as in Equation 2,

$$
T_{g_{p, w}}=\frac{x_{p} \Delta C_{p_{p}}^{a c t} T_{g_{p}}+x_{w} \Delta C_{p_{w}} T_{g_{w}}}{x_{p} \Delta C_{p_{p}}^{a c t}+x_{w} \Delta C_{p_{w}}} \quad \text { Eq. } 2
$$

where $p$ and $w$ refer to polymer and the water diluent, respectively, $x$ refers to the weight or mole fraction and $\Delta C_{\mathrm{p}}$ act is the incremental change in the specific heat at $T_{\mathrm{g}}$ of the units capable of activation. ${ }^{19}$ At water concentrations of $1 \mathrm{wt} \%$ and below, the data tracks predictions quite well. At higher water concentration however, experimentally measured changes are less than predicted. A few comments can be made about the experimental deviations from theory. First, the glass transition resolved from an initial heating of the sample was greater than or equal to that of a subsequent heating of the same sample. This suggests diffusion of water out of the epoxy during the heating profile of the calorimeter does not significantly affect the measurement and cause the deviation from theory predictions. If water was diffusing out of the polymer, the glass transition would 
be anticipated to be higher in the second heating cycle. Diffusion of water out of the polymer at high temperatures would also be anticipated to result in condensation within the sealed sample container upon cooling and a freezing of this condensate at $0^{\circ} \mathrm{C}$. In this testing, samples were cooled to $-50^{\circ} \mathrm{C}$ and no sign of water freezing are noted in the calorimetry data. Second, the 828/DEA mixture is epoxide rich and reactive species are known to exist after the cure profile (as observed from an exothermic peak in the calorimeter upon heating the material above $T_{\mathrm{g}}$ after cure at $\mathrm{T}=70^{\circ} \mathrm{C}$ for 24 hours). The reaction of water with the excess epoxide can proceed to form a glycol unit. In addition, the plasticization of the polymer associated with the water absorption has depressed the $T_{\mathrm{g}}$ to approximately the temperature at which the water imbibition was carried out at, $60^{\circ} \mathrm{C}$. The reduction of $T_{\mathrm{g}}$ to the material temperature increases both the mobility in the polymer and the potential for further polymerization reaction. In principal, the effects of any such reactions on the $T_{\mathrm{g}}$ of the polymer can be resolved by evaluating $T_{\mathrm{g}}$ after removing water from the sample. However, resolving clear conclusions from such experiments has proven challenging. A final experimental comment on this point relates to the potential of physical aging of the sample during the imbibition of water. The water saturated samples were evaluated calorimetrically in a heat-cool-heat temperature ramping scheme between $-50^{\circ} \mathrm{C}$ and $150^{\circ} \mathrm{C}$. Differences are noted in the signatures of the glass transition between the first and second heating cycles. The first heating cycle resolves a higher $T_{\mathrm{g}}$ and an enthalpic peak observed as an "overshoot" of the rubbery response of the material above $T_{\mathrm{g}}$. These are characteristics of physical aging ${ }^{13}$ and are erased upon annealing above $T_{\mathrm{g}}$ and reheating the sample in a second thermal scan. The $T_{\mathrm{g}}$ reported here is that from the second scan, with any such physical aging effects removed. Limitations of the thermodynamics-based theory to capture all aspects of the glass transition process may also exist.

Having established the adhesive $T_{\mathrm{g}}$ depression associated with water sorption and that experimental failure of the virgin napkin-ring joint correlates well with the peak shear stress predicted for the napkin-ring geometry by the SPEC nonlinear viscoelastic model, one might ask whether the change in strength in the napkin-ring joints under humid environments could be predicted by the SPEC model by accounting for the change in $T_{\mathrm{g}}$ of the adhesive. Certainly, some assumptions must be made in order to represent the experimental tests with the model. The principal assumption made here is that the change in $T_{\mathrm{g}}$ of the adhesive can be accounted for in the model by simply shifting the reference temperature (the temperature to which shear relaxation data was shifted in order to construct a master relaxation spectrum for the material by time-temperaturesuperposition), $T_{\text {ref }}$, in the model by the same amount as the $T_{\mathrm{g}}$ depression associated with water sorption. This would imply that the absorption of water does not change the shape of the polymer relaxation spectrum, it only shifts it by a defined amount that can be accounted for by equating a shift in $T_{\text {ref }}$ to the change in the $T_{\mathrm{g}}$. Model predictions of 
napkin-ring response with a range of adhesive reference temperatures are given in Figure 7.
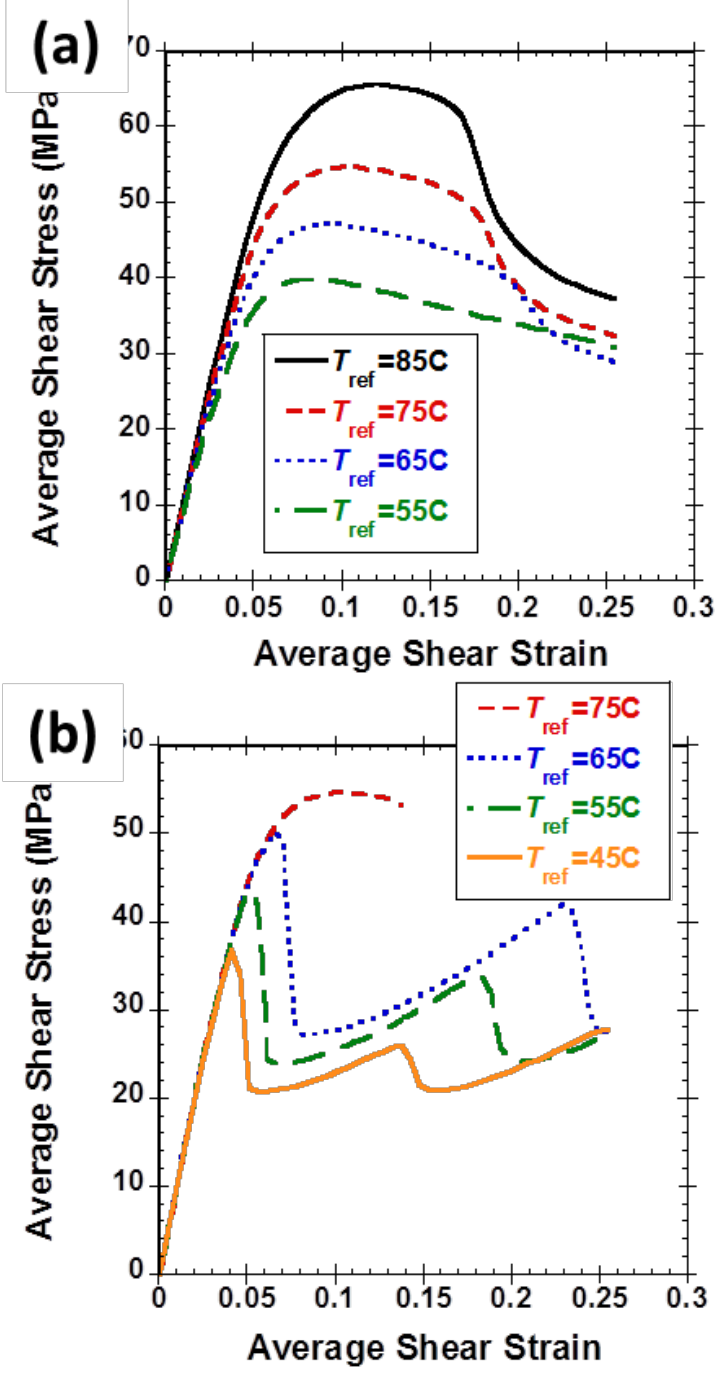

Fig. 7: Finite element model predictions for napkin-ring macroscopic response with change in (a) bulk $T_{\text {ref }}$ and (b) an interfacial $T_{\text {ref }}$ localized to a $10 \mu \mathrm{m}$ layer at both adherend boundaries.

Predictions are for napkin-ring response at $T=23^{\circ} \mathrm{C}$ with the history described in the experimental section.

Both a bulk [Figure 7(a)] and interfacial [Figure 7(b)] change in the $T_{\text {ref }}$ are examined with the model. The latter would be relevant should the water diffuse along the interface at a faster rate than through the bulk. In the case of the interfacial change, $T_{\text {ref }}$ is varied in a $10 \mu \mathrm{m}$ thick interface layer at both adherend boundaries while the $T_{\text {ref }}$ in the bulk adhesive is maintained at $75^{\circ} \mathrm{C}$. While a $10 \mu \mathrm{m}$ interface layer may not be physically 
appropriate for interfacial water diffusion, one may view this as a sensitivity analysis that examines the effect of a non-uniform distribution of polymer mobility through the thickness of the adhesive bondline. Average shear stress in these plots refers to the applied shear force divided by the adhesive-to-adherend bond area. Average shear strain is $\frac{\theta r}{h}$, where $\theta$ is the rotational displacement applied to the napkin-ring, $r$ is the average annular radius, and $h$ is the bondline thickness. As can be seen in Figure 7, changes in

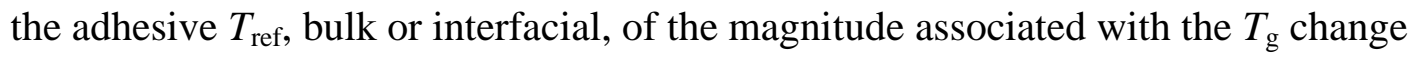
experimentally observed with water absorption into 828/DEA can have a significant effect on the maximum predicted shear stress in the joint. Using the maximum predicted shear stress in the napkin-ring as the failure metric, a comparison can be made between predictions and experimental failure loads. This comparison is given in Figure 8.

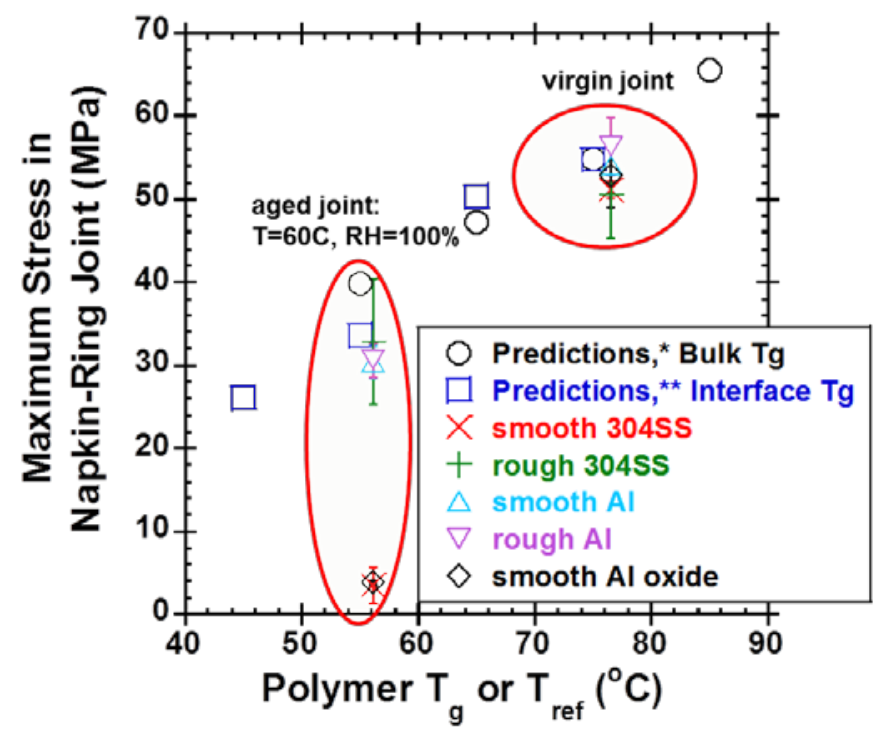

Fig. 8: Maximum stress in napkin-ring joint versus adhesive $T_{\mathrm{g}}$ (experiments) or adhesive $T_{\text {ref }}$ (model predictions).

As already shown, the stress at failure in the virgin joint does not vary significantly amongst adherend type or surface preparation and matches closely to the maximum stress predicted in the joint when modeling the adhesive with the SPEC model. The new information in this plot exists in the comparison of joint strength after equilibration at $T=60^{\circ} \mathrm{C}$ and $\mathrm{RH}=100 \%$ to model predictions. Under these conditions the adhesive $T_{\mathrm{g}}$ has been depressed to approximately 55C and many of the joints exhibit a stress at failure between 30 and $40 \mathrm{MPa}$. This joint strength correlates very closely to the maximum predicted stress in the napkin-ring joint for an adhesive $T_{\text {ref }}$ of $55^{\circ} \mathrm{C}$, be that the bulk or interfacial $T_{\text {ref }}$ of the adhesive. This suggests that the change in joint strength for these 
cases can be accounted for by the reduction in adhesive $T_{\mathrm{g}}$ associated with water sorption. To the best of our knowledge, this is the first time that a predictive capability has been able to account for joint strength depression associated with adhesive $T_{\mathrm{g}}$ depression. There are two joint cases, the smooth 304SS and smooth Al oxide, that exhibit a stress at failure much lower than that predicted to be associated with the adhesive $T_{\mathrm{g}}$ reduction. In these cases, other factors must be accounted for and are of continuing interest to this research.

From Figure 8, it is noted that the effect on the maximum predicted shear stress is different between the bulk and interfacial $T_{\text {ref }}$ changes. This is not surprising and results from the fact that having an interfacial $T_{\text {ref }}$ that varies from the bulk is analogous to bonding the joint with two unique adhesive materials, one sandwiched between the other. The stress in the interface material evolves differently than in the bulk material. This stems from the local strain and shift factor (a measure of the adhesive relaxation rate) of the adhesive varying differently from the top to the bottom of the adhesive bondline thickness for the bulk and interfacial $T_{\text {ref }}$ change scenarios. From Figure 7, it is clear that differences between the bulk and interfacial $T_{\text {ref }}$ changes are not isolated to the local maximum in the stress-strain response. How the local maximum is approached and what occurs after the local maximum are also distinct. For the interfacial $T_{\text {ref }}$ changes, all joint stress-strain responses are equivalent until the local maximum is achieved, whereas that is not the case for the bulk changes in $T_{\text {ref. }}$ The interfacial changes also give a much sharper change in the stress with increasing strain after the local maximum than the bulk changes. Thus, while both scenarios achieve run-away nonlinear viscoelasticity, how they approach this state and how the macroscopic joint responds after the state is reached are clearly different.

Another method to further demonstrate the importance of adhesive $T_{\mathrm{g}}$ depression due to water sorption on the strength of an adhesive joint is to remove the water from the joint and examine whether the strength is rejuvenated. The results of such an experiment are given in Figure 9. 

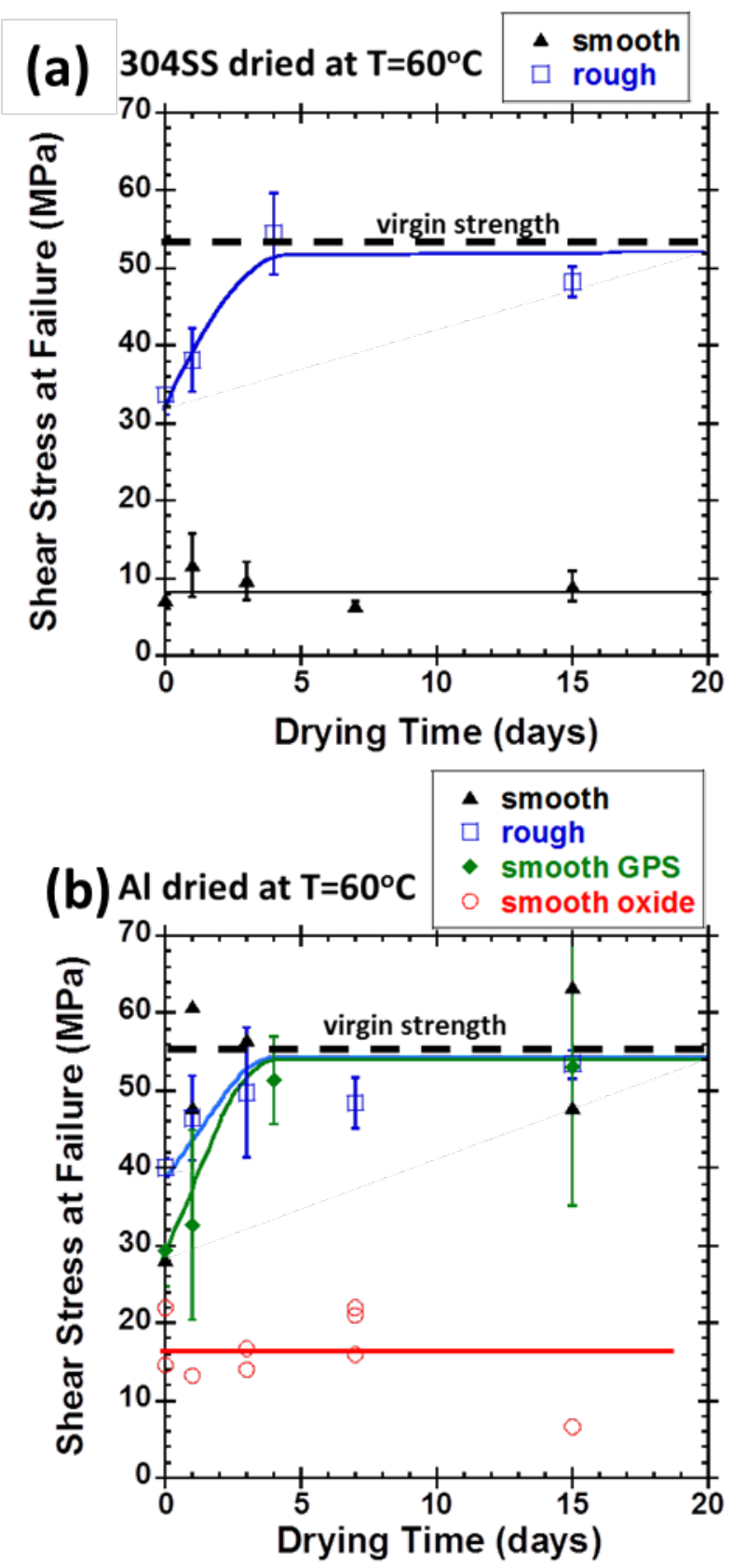

Fig. 9: Napkin-ring shear stress at failure versus time during drying at $T=60^{\circ} \mathrm{C}$ in a dessicated environment (after equilibrating at $T=60^{\circ} \mathrm{C}$ and 100\% RH) for (a) 304SS adherends and (b) Al adherends. The lines are just a guide to the eye. In cases with no error bars, individual data points are plotted rather than averages (points) and standard deviations (error bars). 
From Figure 9, it is observed that in cases where the drop in joint strength can be accounted for by the depression of the adhesive $T_{\mathrm{g}}$, joint strength can be fully rejuvenated to the virgin state upon drying. This observation is not only consistent with the idea that adhesive $T_{\mathrm{g}}$ depression associated with water sorption accounts for the reduction in joint strength (once the adhesive is dried, the virgin $T_{\mathrm{g}}$ is recovered), but it has practical implications. It demonstrates that there are cases where bonds exposed to moisture can just be dried out rather than sacrificed due to fear of bond weakness. Since the details of the adherend composition and surface preparation are important in determining strength recovery, testing the ability to rejuvenate the strength of a particular bond by performing a coupon-level test would be key to defining whether a given structure can be repaired with a drying treatment. On the other hand, in cases where joint strength drops beyond that associated with adhesive glass transition temperature depression, joint strength is not rejuvenated upon drying. Clearly, in these cases another mechanism is at play that affects joint strength in a non-recoverable way, or at least non-recoverable by heating.

The final results reported here are an examination of the role of relative humidity on the strength of the napkin-ring joint at $T=60^{\circ} \mathrm{C}$. Only the smooth $304 \mathrm{SS}$ adherends were examined under these conditions. These adherends result in joints that exhibited the largest observed effect on adhesive strength at $T=60^{\circ} \mathrm{C}$ and $100 \% \mathrm{RH}$ and hence provided the best opportunity to experimentally resolve the role of relative humidity on strength at the same temperature. The results of this examination are given in Figure 10. 

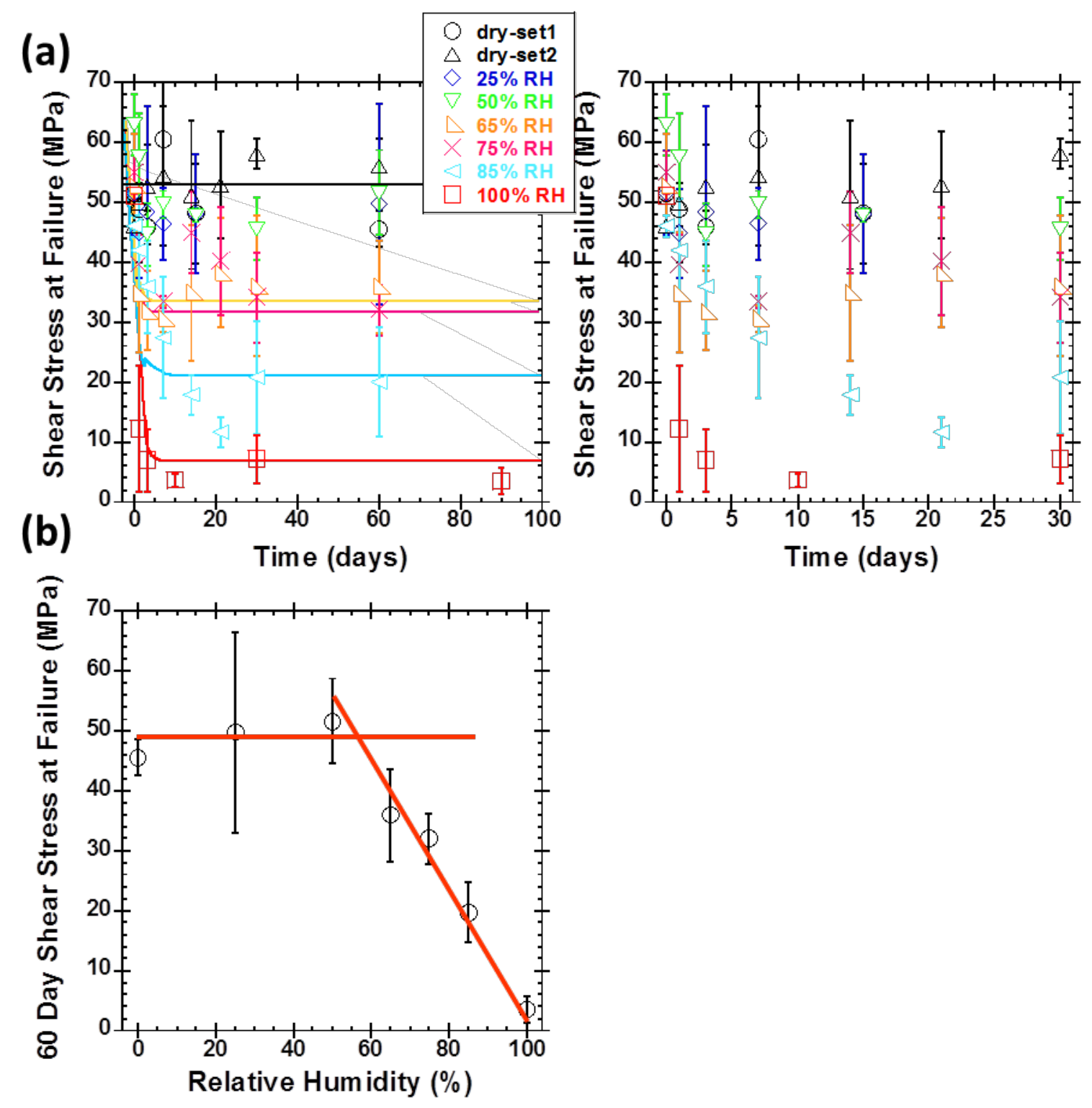

Fig. 10: Smooth 304SS napkin-ring shear stress at failure (a) versus time during exposure to $T=60^{\circ} \mathrm{C}$ and multiple levels of $\mathrm{RH}$ and (b) versus $\mathrm{RH}$ for joints at $T=60^{\circ} \mathrm{C}$ for 60 days. The lines are just a guide to the eye. Plot to the right of (a) illustrates the short time behavior of the data.

From Figure 10(a), there is no clear change in joint shear stress at failure at 50\% RH and below over the 60 day period examined. Certainly, there has been some water sorption into the adhesive and an associated depression of adhesive $T_{\mathrm{g}}$ under these conditions (see Figure 6), but measurements were unable to detect a change in joint strength beyond the experimental uncertainty. Above 50\% RH, the joint strength drops to lower levels with 
time and increasing RH. This is further illustrated in Figure 10(b), in which the shear stress at failure of the joint at 60 days is plotted against relative humidity. The lines in Figure 10(b), drawn only as a guide to the eye, are suggestive of a critical humidity, below which adhesive strength does not degrade. The existence of such a critical humidity, or potentially a critical water concentration within the adhesive, has been suggested in previous works. ${ }^{11,20}$ Here, the critical humidity is suspected to be associated with a process that goes on at the adhesive-304SS interface and ultimately reduces the joint strength beyond that associated with water absorption within the adhesive alone. Identification of this process is the focus of current investigations.

\section{Conclusions}

In summary, a number of points will be re-emphasized. First, while adherend composition and surface preparation did not have a significant effect on virgin joint debonding stress, these factors do significantly impact the role of moisture on the strength of the joint. For the 304SS adherends, surface abrasion appears to be a defining factor in determining the effect of moisture on the joint strength. The red garnet blasted surfaces demonstrated a significant increase in the steady-state strength of the joint upon exposure to $\mathrm{T}=60 \mathrm{C}$ and $100 \% \mathrm{RH}$, compared to the polished and bead blasted surfaces. The hypothesis currently under investigation to explain this behavior is that an interface specific process that occurs at the polished and bead blasted surfaces is no longer active after red garnet blasting. For $\mathrm{Al}$ adherends, surface abrasion appears to play less of a role in determining the effect of moisture on joint strength, and the "corrosion" of the bonding interface is what drives joint strength to decrease below that predicted based on adhesive $T_{\mathrm{g}}$ depression associated with water absorption. Interestingly, the addition of primer or silane coupling agent did not result in significant changes in behavior for either adherend.

Considering the correlation between joint failure and SPEC model predictions of maximum shear stress in the napkin-ring, the mechanism of failure in the virgin joint is thought to be run-away nonlinear viscoelasticity in the polymer adhesive (i.e., cohesive failure in the adhesive). Changes in adherend composition and surface preparation do not change the locus of failure in the virgin joint and hence joint strength is always defined by the cohesive strength of the polymer. When depression of joint strength in humid environments is fully accounted for by depression of the adhesive $T_{\mathrm{g}}$ due to water absorption, failure remains cohesive in the polymer and joint strength can be rejuvenated by drying. However, when joint strength falls below this level, other surface specific phenomena must change the locus of failure to the interface. Understanding what the surface specific phenomena are and how they affect interfacial failure are of interest and continue to be investigated. At this point, these failure mechanisms cannot be accounted 
for in a predictive technique. More details of specific mechanisms are necessary. The experimentation on joint drying and $\mathrm{RH}$ levels do provide some information on the surface specific effects. For instance, the process active in smooth 304SS joints at $T=60^{\circ} \mathrm{C}$ and $100 \% \mathrm{RH}$ is not reversible upon drying, as observed by the inability to recover joint strength. It also appears that $\mathrm{RH}$ must be greater than $50 \%$ for the process to occur within 60 days. Unfortunately, these observations cannot pinpoint a specific mechanism. They can only serve to help eliminate some possibilities.

A physically-based path toward predicting the degradation in adhesive strength associated with water sorption into the polymer adhesive for geometries that do not involve severe strain gradients can be envisioned. A model of water influx into the polymer adhesive could be coupled with a NLVE representation of the polymer response to predict run-away nonlinear viscoelasticity. The plasticization and swelling effects of the water on the adhesive must be accounted for, and this may be possible in SPEC in an analogous manner as done to incorporate cure effects. Rigorously coupling diffusion and constitutive response for the multicomponent viscoelastic system may be more complicated. Alternatively, given water diffusion rates and the effect of water on the $T_{\mathrm{g}}$ of the adhesive, maybe an engineering approach would be simpler and sufficient. The best method to examine this would be to work the problem in a rigorous manner and then evaluate where simplifications can be made without losing fidelity in the predictions. We hope to explore this approach in coming years.

\section{Acknowledgements}

JMK thanks E.D. Reedy for helpful discussions on this manuscript and Rex Jaramillo for sample preparation help. Sandia National Laboratories is a multi-program laboratory managed and operated by Sandia Corporation, a wholly owned subsidiary of Lockheed Martin Corporation, for the U.S. Department of Energy’s National Nuclear Security Administration under contract DE-AC04-94AL85000.

\section{Appendix}

Glassy polymers exhibit complex nonlinear, time-dependent relaxations in stress and volume. This has led to the development of a number of constitutive equations based on the assumption of thermorheological simplicity and the use of a material clock. These clock models have been driven by a variety of different mechanisms such as free volume, entropy, stress and strain. Caruthers et al. ${ }^{3}$ introduced a rigorous potential energy clock (PEC) model based on a Frechet series expansion of the Helmholtz free energy for materials with fading memory. This formulation enabled the clock to be based on readily computable thermodynamic quantities, which proved to be quite successful in quantitatively predicting a wide range of material behavior (e.g., stress relaxation, physical aging, creep, yielding) from a single set of model parameters. Unfortunately, the PEC constitutive equations are quite complex involving four relaxation spectra, single 
and double hereditary integrals and a variety of temperature and volume dependencies. The simplified potential energy clock (SPEC) model was developed to ease the experimental and computational burdens of PEC. Although SPEC is a more phenomenological model that is easier to characterize and calibrate, its performance predictions have not been compromised. ${ }^{11}$

For infinitesimal deformations, the SPEC viscoelastic stress equations can be written as

$$
\begin{aligned}
\underline{\sigma}(t)= & \left.\left\{K_{g}-K_{e q}\right\} \int_{0}^{t} d s f_{1}\left(t^{*}-s^{*}\right) \frac{d I_{1}}{d s}(s)\right] \underline{\underline{I}} \\
& -\left[\left\{K_{g} \beta_{g}-K_{e q} \beta_{e q}\right\} \int_{0}^{t} d s f_{1}\left(t^{*}-s^{*}\right) \frac{d T}{d s}(s)\right] \underline{\underline{I}} \\
& +2\left\{G_{g}-G_{e q}\right\} \int_{0}^{t} d s f_{2}\left(t^{*}-s^{*}\right) \frac{d \underline{\varepsilon}_{d e v}}{d s}(s)+ \\
& +\left[K_{e q}\left\{I_{1}(t)-\beta_{e q}(T(t)-T(0))\right\}\right] \underline{\underline{I}}+2 G_{e q} \underline{\underline{\varepsilon}}_{d e v}(t)
\end{aligned}
$$

where $I_{1}=\underline{\underline{I}}: \underline{\underline{\varepsilon}}, \underline{\underline{\varepsilon}}_{d e v}=\underline{\underline{\varepsilon}}-\frac{1}{3} I_{1} \underline{\underline{I}}, \beta$ is the volumetric thermal expansion coefficient and the subscripts "g” and "eq" denote glassy and equilibrium quantities. The strain measure, $\underline{\underline{\varepsilon}}$, is defined as the integral of the rate of deformation tensor, $\underline{\underline{\sigma}}$ is the Cauchy stress, $K$ and $G$ are the bulk and shear moduli, $T$ is temperature. The relaxation functions, $f_{1}(t)$ and $f_{2}(t)$, decay from one to zero and are defined from an exponential series expansion in time (i.e., a Prony series) characterized at a reference temperature, $T_{r e f}$, adopted for all properties. In general, all the integral prefactors can depend on temperature, in which case a Taylor series expansion about the reference temperature state is used to define the function. For example, $K_{g}(T)=K_{g_{-} \text {ref }}+\left(d K_{g} / d T\right)\left(T-T_{\text {ref }}\right)$. The material clock definition is based on a shift factor, a, that is a function of the thermal, volumetric and deviatoric deformation histories as follows:

$$
\begin{gathered}
t^{*}-s^{*}=\int_{0}^{t} \frac{d w}{a(w)} \\
\log (a(t))=-\frac{C_{1} N(t)}{\left(C_{2}+N(t)\right)}
\end{gathered}
$$




$$
\begin{aligned}
N(t)= & \left\{\left[T(t)-T_{r e f}\right]-\int_{0}^{t} d s f_{1}\left(t^{*}-s^{*}\right) \frac{d T}{d s}(s)\right\} \\
& +C_{3}\left\{I_{1}(t)-\int_{0}^{t} d s f_{1}\left(t^{*}-s^{*}\right) \frac{d I_{1}}{d s}(s)\right\} \\
& +C_{4}\left\{\int_{0}^{t} d s f_{1}\left(t^{*}-s^{*}\right) \frac{d \underline{\varepsilon}_{d e v}}{d s}(s)\right\}:\left\{\int_{0}^{t} d s f_{1}\left(t^{*}-s^{*}\right) \frac{d \underline{\varepsilon}_{d e v}}{d s}(s)\right\}
\end{aligned}
$$

The quantity $\mathrm{N}$ is an approximation to the potential internal energy that drives the relaxation rates through the material clock. ${ }^{11}$ The parameters $\mathrm{C}_{1}$ through $\mathrm{C}_{4}$ are clock constants that must be fit to data. The first two parameters are related to the WilliamsLandel-Ferry (WLF) coefficients, and the third constant controls the pressure dependence of the glass transition temperature. The $\mathrm{C}_{4}$ coefficient multiplies a second invariant of the effective deviatoric, viscoelastic strain history. It establishes the dependence of the material clock on the mechanical deformation and is used to drive polymer "yielding". For modeling the linear viscoelasticity, $\mathrm{C}_{3}$ and $\mathrm{C}_{4}$ are set to zero. Further details describing the derivation of these equations can be found in references. ${ }^{3,11}$ 


\section{References}

1. Reedy, E. D., Jr.; Guess, T. R. J. Adhes. Sci. Technol. 1996, 10, (1), 33-45.

2. Adolf, D. B.; Chambers, R. S.; Hance, B.; Elisberg, B. J. Adhes. 2010, 86, (11), 1111-1131.

3. Caruthers, J. M.; Adolf, D. B.; Chambers, R. S.; Shrikhande, P. Polymer 2004, 45, (13), 4577-4597.

4. Kinloch, A. J., Adhesion and Adhesives. Chapman and Hall: 1987; p 454 pp.

5. Brown, H. R. Annu. Rev. Mater. Sci. 1991, 21, 463-89.

6. Adolf, D. B.; Chambers, R. S.; Stavig, M. E.; Kawaguchi, S. T. J. Adhes. 2006, 82, (1), 63-92.

7. Reedy, E. D., Jr.; Guess, T. R. J. Adhes. Sci. Technol. 1995, 9, (2), 237-51.

8. Bikerman, J. J., The Science of Adhesive Joints. 2nd ed. Academic: 1968; p 349 pp.

9. Silva, L. F. M.; Sato, C., Design of Adhesive Joints under Humid Conditions. SpringerVerlag: Berlin, 2013; Vol. 25 of Advanced Structured Materials Series. See Chapters 7 and 8 for work focused on coupling moisture diffusion and mechanical response of adhesive joints using a progressive damage approach.

10. Adolf, D. B.; Chambers, R. S.; Neidigk, M. A. Polymer 2009, 50, (17), 4257-4269. See Appendix for a brief summary of key aspects of the SPEC NLVE model.

11. Brewis, D. M.; Comyn, J.; Raval, A. K.; Kinloch, A. J. Int. J. Adhes. Adhes. 1990, 10, (4), 247-53.

12. Kinloch, A. J. J. Mater. Sci. 1980, 15, (9), 2141-66.

13. Struik, L. C. E., Physical Aging in Amorphous Polymers and Other Materials. Elsevier Scientific Publishing Co.: 1978; p 230 pp.

14. Adolf, D. B., Predicting Stresses in Thermosets. Sandia National Laboratories: Albuquerque, 2010; p 144.

15. Venables, J. D. J. Mater. Sci. 1984, 19, (8), 2431-53.

16. Ellis, T. S.; Karasz, F. E. Polymer 1984, 25, (5), 664-9.

17. Crank, J., The Mathematics of Diffusion. 2d Ed. Oxford Univ. Press: 1975; p 414 pp.

18. Maggana, C.; Pissis, P. J. Polym. Sci., Part B: Polym. Phys. 1999, 37, (11), 11651182.

19. Ellis, T. S.; Karasz, F. E.; ten Brinke, G. J. Appl. Polym. Sci. 1983, 28, (1), 23-32.

20. Cognard, J. Int. J. Adhes. Adhes. 1988, 8, (2), 93-9. 